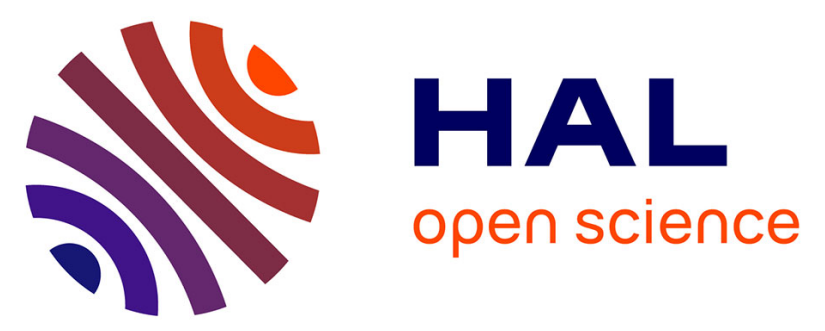

\title{
Femtosecond laser structuring of silver-containing glass: Silver redistribution, selective etching, and surface topology engineering
}

Jean-Charles Desmoulin, Yannick Petit, Lionel Canioni, Marc Dussauze, Michel Lahaye, Hernando Magallanes Gonzalez, Etienne Brasselet, Thierry Cardinal

\section{To cite this version:}

Jean-Charles Desmoulin, Yannick Petit, Lionel Canioni, Marc Dussauze, Michel Lahaye, et al.. Femtosecond laser structuring of silver-containing glass: Silver redistribution, selective etching, and surface topology engineering. Journal of Applied Physics, 2015, 118 (21), 213104 (7 p.). 10.1063/1.4936233 . hal-01243619

\section{HAL Id: hal-01243619 \\ https://hal.science/hal-01243619}

Submitted on 23 Feb 2016

HAL is a multi-disciplinary open access archive for the deposit and dissemination of scientific research documents, whether they are published or not. The documents may come from teaching and research institutions in France or abroad, or from public or private research centers.
L'archive ouverte pluridisciplinaire HAL, est destinée au dépôt et à la diffusion de documents scientifiques de niveau recherche, publiés ou non, émanant des établissements d'enseignement et de recherche français ou étrangers, des laboratoires publics ou privés.

\section{다(1)(2)}

Distributed under a Creative Commons Attribution - ShareAlikel 4.0 International 


\title{
Femtosecond laser structuring of silver-containing glass: Silver redistribution, selective etching, and surface topology engineering
}

\author{
Jean-Charles Desmoulin, ${ }^{1}$ Yannick Petit, ${ }^{1}$ Lionel Canioni, ${ }^{2}$ Marc Dussauze, ${ }^{3}$ \\ Michel Lahaye, ${ }^{4}$ Hernando Magallanes Gonzalez, ${ }^{5}$ Etienne Brasselet, ${ }^{5}$ \\ and Thierry Cardinal ${ }^{1, a)}$ \\ ${ }^{1}$ CNRS, ICMCB, UPR 9048, F 33600 Pessac, France and Univ. Bordeaux, ICMCB, UPR 9048, \\ F 33600 Pessac, France \\ ${ }^{2}$ Université Bordeaux, Centre Lasers Intenses et Applications UMR 5107 CNRS, 351 cours de la Libération, \\ 33405 Talence Cedex, France \\ ${ }^{3}$ Université de Bordeaux, Institut des Sciences Moléculaires, CNRS UMR 5255, 351 cours de la Libération, \\ 33405 Talence Cedex, France \\ ${ }^{4}$ Université de Bordeaux, Placamat, avenue Docteur Albert Schweitzer, 33608 Pessac Cedex, France \\ ${ }^{5}$ Université Bordeaux, Laboratoire Ondes et Matière d'Aquitaine UMR 5798, CNRS, \\ 351 cours de la Libération, 33405 Talence Cedex, France
}

Femtosecond direct laser writing in silver-containing phosphate glasses allows for the threedimensional (3D) implementation of complex photonic structures. Sample translation along or perpendicular to the direction of the beam propagation has been performed, which led to the permanent formation of fluorescent structures, either corresponding to a tubular shape or to two parallel planes at the vicinity of the interaction voxel, respectively. These optical features are related to significant modifications of the local material chemistry. Indeed, silver depletion areas with a diameter below $200 \mathrm{~nm}$ were evidenced at the center of the photo-produced structures while photo-produced luminescence properties are attributed to the formation of silver clusters around the multiphoton interaction voxel. The laser-triggered oxidation-reduction processes and the associated photo-induced silver redistribution are proposed to be at the origin of the observed original 3D luminescent structures. Thanks to such material structuring, surface engineering has been also demonstrated. Selective surface chemical etching of the glass has been obtained subsequently to laser writing at the location of the photo-produced structures, revealing features with nanometric depth profiles and radial dimensions strongly related to the spatial distributions of the silver clusters.

\section{INTRODUCTION}

Direct Laser Writing (DLW) in transparent materials has been investigated for the two last decades. Since the controlled index variation demonstrated by Davis et al., ${ }^{1}$ multiple achievements have been obtained such as nano-gratings. ${ }^{2}$ In addition to the development of laser sources, the engineering of material compositions makes it possible to take advantage of phenomena such as oxido-reduction, diffusion, aggregation, or phase transition processes to access innovative approaches for multi-scale material structuring. Glassy materials are excellent candidates for DLW since they are cost-effective materials and offer very good optical properties in the visible and near infrared (NIR) where lie most of the main photonic applications. The advantage of using short pulse laser is mainly the access to nonlinear absorption processes which guaranty a three-dimensional (3D) structuring in transparent materials hardly feasible with other techniques.

This DLW approach is based on the perfect control of the physico-chemical phenomena and the choice of the appropriate wavelength, pulse duration, energy, and repetition rate. High repetition laser can allow for the control of

\footnotetext{
a) Author to whom correspondence should be addressed. Electronic mail: thierry.cardinal@icmcb.cnrs.fr
}

the heat accumulation, for the monitoring of ionic migration and if eventually subsequent phase transition. Then the local precipitation of crystals within the glass or the local modification of the chemical composition distribution around the laser voxel become accessible. ${ }^{3,4}$ The combination of the DLW and chemical etching has been also developed in order to design innovative topology and micro-channels. Such phenomena combine photo-chemistry, imprinted stress, and selective surface reaction, as demonstrated, for instance, in silica. $^{5,6}$

Our group has shown that the laser structuring of silvercontaining phosphate glass with high repetition rate nearinfrared femtosecond sources resulted in the fabrication of fluorescent pipes that correlatively exhibit an electric field induced second harmonic generation (EFISHG) response. ${ }^{7}$ The structure originates from the multiphoton generation of free electrons, electron diffusion, and trapping, thermal and thermally activated species diffusions leading to the creation of a space charge separation and to the reduction of silver ions into silver clusters exhibiting visible luminescence at the surrounding of the interaction voxel. ${ }^{8}$ It has been evidenced that the charge separation, corresponding to the formation of a perennial static electric field with radial distribution, appears in the early stages of laser irradiation 
for lower deposited dose while the cluster formation requires larger doses.

In this study, physico-chemical characterization has been performed in order to clarify the materials evolution and to demonstrate the role of ionic migration. This material modification has been exploited in order to pattern the material surface thanks to structures imprinted in glass volume and at the surface by the femtosecond laser process associated with chemical etching.

\section{EXPERIMENTAL}

A $0.4 \mathrm{P}_{2} \mathrm{O}_{5} \quad 0.55 \mathrm{ZnO}-0.01 \mathrm{Ga}_{2} \mathrm{O}_{3} \quad 0.04 \mathrm{Ag}_{2} \mathrm{O}$ glass was elaborated from reagents $\mathrm{Zn}\left(\mathrm{PO}_{3}\right)_{2}, \mathrm{ZnO}, \mathrm{Ga}_{2} \mathrm{O}_{3}$, and $\mathrm{Ag}\left(\mathrm{NO}_{3}\right)$. Samples were fabricated using a classical meltquenching technique. Melting was conducted in a platinum crucible at $1200^{\circ} \mathrm{C}$ during $12 \mathrm{~h}$. In order to improve the homogeneity of the glass, the sample was quenched, ground and melted twice. Then, the samples were annealed at $40^{\circ} \mathrm{C}$ below the glass transition $\left(\mathrm{Tg}=370^{\circ} \mathrm{C}\right)$ for $4 \mathrm{~h}$.

Direct laser writing was performed thanks to a home made microscope combining a NIR femtosecond laser (KGW: $\mathrm{Yb}$ oscillator, $0.6 \mathrm{~V}, 10 \mathrm{MHz}$ at $1030 \mathrm{~nm}$ ), a microscope objective $(20 \times$, NA 0.4$)$, and a high-precision 3D translation stage XMS-50 (50 nm resolution). Photo-induced structures were produced $160 \mu \mathrm{m}$ below the sample surface by translating the sample along or perpendicularly to the propagation axis of the laser beam at a controlled speed ranging from $10 \mu \mathrm{m} / \mathrm{s}$ to $50 \mu \mathrm{m} / \mathrm{s}$.

Chemical analysis was conducted by electron microprobe (Wavelength Dispersive Spectroscopy (WDS)) CAMECA SX 100. Scans were performed with the following conditions: $15.1 \mathrm{keV}, 9.9734 \mathrm{nA}$, dwell time $=0.2 \mathrm{~s}$, step size $=119 \mathrm{~nm}$. Prior local chemical analysis, the sample had been polished orthogonally to the written tubes' axis in order to bring the corresponding section to the sample surface (corresponding to linear structures obtained by translating the sample parallel to the beam axis). The same prior preparation was applied for the double plan structures (corresponding to linear structures obtained by translating the sample perpendicularly to the beam axis).

Soft chemical etching was realized in de-ionized (DI) water for $26 \mathrm{~min}$ to engineer the local topology of few tens of nanometers. Topology analysis was conducted using atomic force microscopy (AFM) by a Bruker Dimension Icon in KPFM mode (Kelvin Probe Force Microscope). Glass surface has been immerged in demineralized water prior investigation.

Spatial measurements of fluorescence were made thanks to a Leica DM6000 TCS SP8 X confocal microscope. An oil-immersion objective $63 \times$ was used. Excitation wavelength was $405 \mathrm{~nm}$, and emission wavelength was filtered on a bandwidth of $50 \mathrm{~nm}$ centered on $450 \mathrm{~nm}$.

\section{RESULTS AND DISCUSSION}

\section{DLW of luminescent structures}

$3 \mathrm{D}$ structures were written in the volume (typically $160 \mu \mathrm{m}$ under the surface) of a silver-containing phosphate glass thanks to a focused femtosecond laser beam as previously reported. ${ }^{9}$ Thanks to the nonlinear optical absorption, local structuring occurs in the voxel of interaction volume. It was demonstrated that electrons and holes are trapped in the first step, locally creating a string and perennial electric field. ${ }^{10}$ The high repetition rate of the femtosecond pulsed laser induces a thermally activated silver ion migration. This phenomenon is then developed pulse after pulse by heat accumulation, which results in the aggregation of silver atomic and ionic species. ${ }^{11}$ The process of ion migration has been proposed to occur radially from the center of the beam to the edge. ${ }^{12}$ The combination of electrons production, silver reduction, and silver migration leads to the permanent formation of luminescent $\mathrm{Ag}_{\mathrm{m}}{ }^{\mathrm{x}+}$ clusters at the surrounding of the voxel. Sample motion during the laser writing process gives access to the formation of 3D fluorescent structures with an excitation ranging from $270 \mathrm{~nm}$ to $530 \mathrm{~nm}$ and a broad emission covering the whole visible range. This original luminescence property is located on the edge of the volume of interaction, as shown in Figure 1, leading to the formation either of a tubular structure or of a two parallel planes structure, for sample motion along or perpendicular to
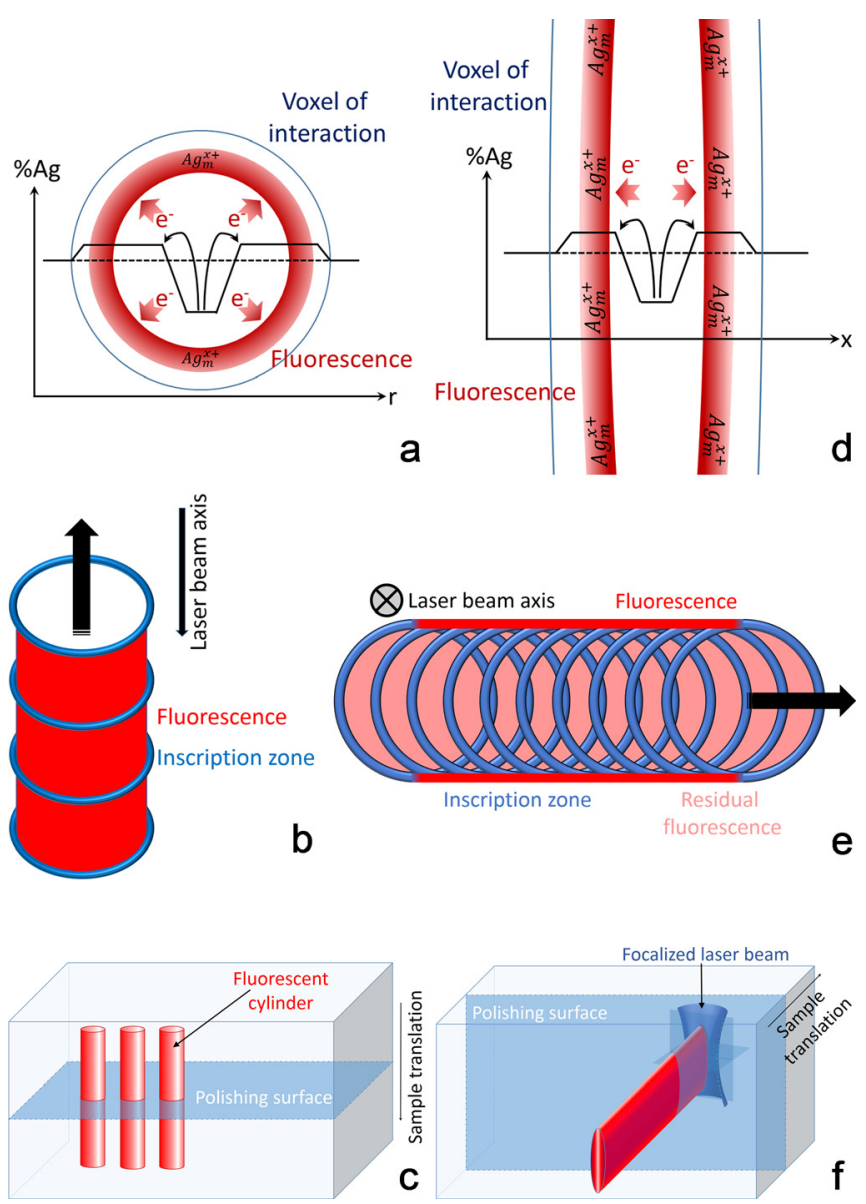

FIG. 1. Top view of the diameter (a) and side view of the confocal parame ter trace (d) of the interaction voxel, and fluorescent silver cluster location resulting from electron trapping combined with silver ion migration; description of the cumulative overlaying of the different pulse to pulse vox els for linear motion longitudinally (b) and perpendicularly (e) to the laser propagation axis, respectively; scheme of the DLW procedure for sample motions along (c) and perpendicular (f) to the laser propagation axis, result ing in the observed fluorescence location. 
the laser beam propagation axis, respectively. The size of this structure is fixed by the voxel parameters and by chemical diffusion properties of the glass, as shown in Figures 1(a) and $1(\mathrm{~d})$.

The diameter of the cylindrical structure typically corresponds to the beam diameter of $2.4 \mu \mathrm{m}$, and the depth of the double plan structure corresponds to the confocal parameter of about $12 \mu \mathrm{m}$. This photo-induced phenomenon is dependent on irradiance, number of pulses, laser repetition, and sample motion speed as reported by Bellec et al. ${ }^{13}$

\section{Characterization of the luminescent structure}

Figure 2(a) corresponds to the reconstruction of the fluorescent complex structures with a cylindrical shape obtained after collecting fluorescence confocal microscope images of the section of the structure, for an excitation at $405 \mathrm{~nm}$ and an emission centered at $450 \mathrm{~nm}$ (Figure 2(b)). In the case of the motion perpendicular to the laser propagation axis, the resulting luminescent structure corresponds to two parallel luminescent surfaces. Figure 2(c) corresponds to the fluorescence intensity profile of the section of the cylindrical structure. Figure $2(\mathrm{~d})$ is a top view of the fluorescent structure while Figure 2(e) represents the view of the section of the fluorescent structure. Figure 2(f) represents the luminescence
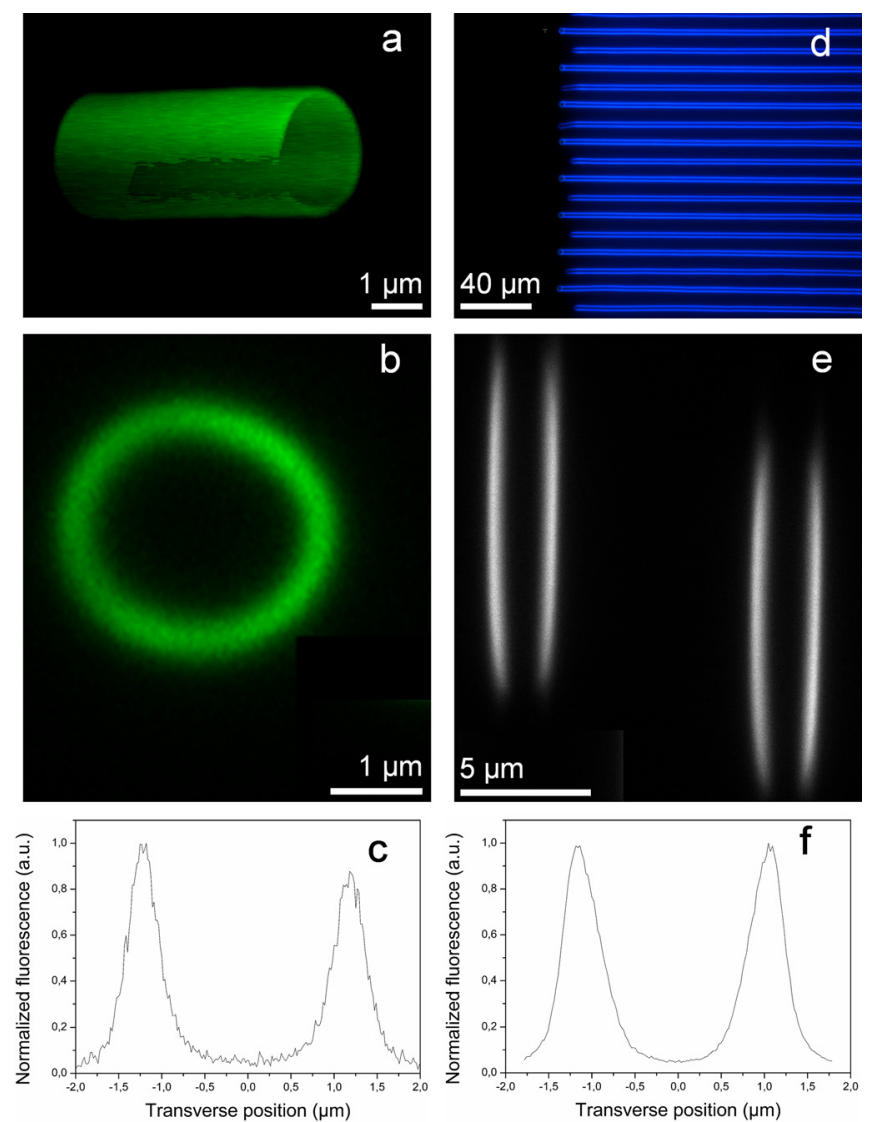

FIG. 2. Confocal fluorescence imaging of a cylindrical structure (a), of the cross section of a cylindrical structure (b), of the top view of a series of dou ble plane structures (d), of the cross section of double plane structures (e). Fluorescence intensity distribution of the cross sections of laser induced structures for a sample motion perpendicular (c) and parallel (f) to the laser beam propagation axis. intensity profile across the double plane structure. In the particular case of the perpendicular motion of the sample, erasure phenomenon occurs. During the sample motion, the silver clusters formed at the front of the laser beam along its track are progressively illuminated by the center of the Gaussian beam profile where lies the maximum irradiance (Figure 1(e)), which results in the dissolution of the cluster. The fluorescent photoproduced features only remain at the border of the laser track, where not sufficiently high irradiances are experienced, which prevents the photo-dissociation of the previously photoinduced silver clusters, leading to the observed double line structures. Contrarily to the motion along the beam propagation axis, the overlaying of the successive interaction volume for the motion perpendicular to the beam propagation axis does not preserve the initial cylindrical laser-induced geometry. The geometry of the resulting fluorescent structure thus corresponds to a two parallel planes structure with a depth of $12 \mu \mathrm{m}$ corresponding to the confocal parameter spaced by the beam waist parameter of $2.15 \mu \mathrm{m}$. The length of the plane along the axis of motion is directly corresponding to the displacement of the sample as shown in Figure 2(d). The fluorescence structure across a plane showed a full width at half maximum (FWHM) of $0.5 \mu \mathrm{m}$ (Figure 2).

Motion speeds of $50 \mu \mathrm{m} / \mathrm{s}$ and $10 \mu \mathrm{m} / \mathrm{s}$ for sample motions along and perpendicular to the beam propagation axis, respectively, have been selected in order to obtain a similar number of overlaid pulses, leading to a comparable deposited dose during the writing procedure. Indeed, motion along the propagation axis insures a larger overlaying of the interaction zones during the sample motion, taking into account the larger size of the confocal parameter as compared to the beam waist.

The corrected emission spectra of the structure have been collected by confocal microscopy. For both sample motions, for excitation at $325 \mathrm{~nm}$, a broad visible emission from $400 \mathrm{~nm}$ to $700 \mathrm{~nm}$ can be observed. Such emission is in accordance with the formation of silver clusters $\mathrm{Ag}_{\mathrm{m}}{ }^{\mathrm{x}+}$, as previously reported. ${ }^{14}$

\section{Chemical analysis within the photo-induced structure}

The chemical composition variation across the photoproduced structures has been investigated by WDS and statistically reported thanks to repeated measurements on 16 photo-produced structures. The analysis has shown that the ratio of gallium, phosphorus, and zinc elements does not evidence significant variation along the transverse section of the photo-induced structures. The evolution, if any, remains below the detection accuracy of the instrument. Only the silver content exhibits a significant distribution modification from the nominal composition of the glass in the irradiated area. The relative concentration of silver, as compared to other constituents of the glass, decreases for both sample motions in the center of the structure where the laser energy deposition is maximal. The measurement was not influenced by the induced topology (at maximum $250 \mathrm{~nm}$ ), knowing that the experimental volume of the probe is $1 \mu \mathrm{m}^{3}$ due to the pear shape of the electron beam after multiple scattering broadening while penetrating the glass material within a few 
microns. The theoretical atomic percentage of silver in the glass is $7.64 \%$, which is verified in pristine zones. The concentration profiles along the photo-induced structures are shown in Figure 3 in the case of annular patterns. For such a sample translation along the beam propagation axis, a clear depletion of the silver can be observed with a decrease of about 10 at. \% of silver at the very center of the structure.

\section{The measured profile of the depletion is not uniform}

A sharp decrease of the silver concentration can be observed in the center and can be described by a Lorentz distribution, corresponding to the response from the surface of the structured material, typically from material depths of a few tens of nanometers. This Lorentz shape is superposed with a broader Gaussian shaped distribution. The estimated Lorentzian and Gaussian widths (FWHM) are $0.13 \mu \mathrm{m}$ and $1.38 \mu \mathrm{m}$, respectively.

The chemical measurement is the convolution between the instrumental response and a real silver depletion distribution. Therefore, the pear-shaped interaction volume of the electron beam has been taken into account in terms of the distribution of intensity, space distribution, and diffusion processes in the glass in order to estimate the real depletion profile.

Experiments allowing for the characterization of the electron beam spatial extension of the microprobe have been conducted. The interaction of the secondary electron beam with a gold sample with extremely sharp step-like edges, deposited on a graphite substrate, led to the validation that the spatial resolution of the SEM probe is typically $0.10 \mu \mathrm{m}$. This measurement confirms that the observed narrow silver depletion with FWHM of $0.13 \mu \mathrm{m}$ (Lorentzian shape at the center), exactly measured in the central region of the photo-induced structures with a systematic repeatability, does not correspond to an artifact, but to a realistic silver depletion. According to the spatial resolution of the apparatus and the spatial measurement sampling of $0.12 \mu \mathrm{m}$ size, the real narrow silver depletion is understood as being narrower than the observed Lorentzian distribution fitted to $0.13 \mu \mathrm{m}$ (FWHM). Regarding the broad

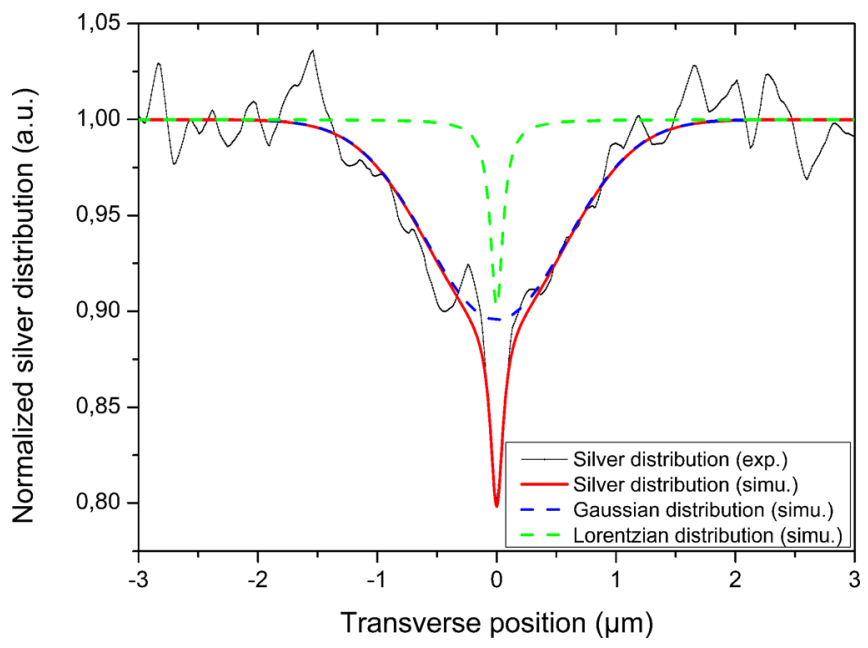

FIG. 3. Silver distribution along the cross section of a pipe structure obtained by WDS microprobe (black curve). This curve is fitted thanks to the sum of a Gaussian curve (dashed blue curve) and a Lorentzian curve (dashed green curve).
Gaussian silver depletion signal that can be observed below the sharp central depletion, as shown in Figure 3, Monte Carlo simulations highly suggested that the electronic beam profile undergoes a strong spatial broadening, due to multiple scattering of the injected electrons within the first microns below surface, leading to an effective broad Gaussian-shaped electron beam, while the Lorentzian-shaped signal corresponds to the near-surface response of the material, typically from the first tens on nanometers below the glass surface. Therefore, the Gaussian-shaped apparent depletion has most probably no physical meaning with respect to the silver distribution. Indeed, it is related to the electron/matter pear-shaped interaction volume, especially originating from the convolution of the Gaussian shaped electron beam (FWHM more than $1 \mu \mathrm{m}$ ) deep below the glass surface (from $100 \mathrm{~nm}$ to $2 \mu \mathrm{m}$, typically) with the strong and much narrower silver depletion (FWHM less than $0.1 \mu \mathrm{m}$ ) along the central axis of the photoinduced semi-infinite cylindrical structure. The origin of the narrow silver central depletion has not been elucidated to date since it is much narrower than the four-photon absorption process responsible for the ionization phenomena. ${ }^{14}$

In the case of a perpendicular motion of the sample, the depletion is corresponding to a decrease of 4 at. \%, which is much lower and therefore more difficult to evidence clearly since this value is close to the measurement error bars. Nevertheless, those experiments validate the silver migration from the center region to the edge of the structure during the DLW procedure. The results contribute to validate the hypothesis of the thermal accumulation process thanks to the high repetition rate of the laser inducing motion of silver from the center to the edge of the interaction voxel.

No clear evidence of silver concentration increase in the location of the fluorescent ring is observed. Taking into account the volume facing the silver depletion and the volume concerned by the presumed silver accumulation, it appears, for instance, that in the case of a cylindrical structure, the volume of depletion is at least 4 times smaller than the volume of the fluorescent shell where the silver accumulation is expected to occur. According to the accuracy of the $\mathrm{X}$-ray microprobe techniques, it becomes then difficult to detect with any certainty an accumulation of silver ions or atoms of about 23 at. \%.

One has to mention that, for both sample motions parallel or perpendicular to the beam propagation axis, there is no significant luminescence at the center of the structure for an excitation at $405 \mathrm{~nm}$. This phenomenon is attributed to the silver cluster dissolution and to the silver reservoir depletion in the center location of the beam for the considered high irradiances. In the particular case of the perpendicular sample motion, these effects are also responsible for the absence of cluster formation behind the laser track. Indeed, during the sample displacement, the central zone that has experimented high irradiance and silver depletion is progressively located on the back edge of the voxel. At this location behind, a new apparent writing threshold (higher threshold) appears near the central zone due to the previous silver depletion which does not allow for the new silver cluster formation behind the beam track. In other words, a higher dose is needed to write again as the local concentration of silver 


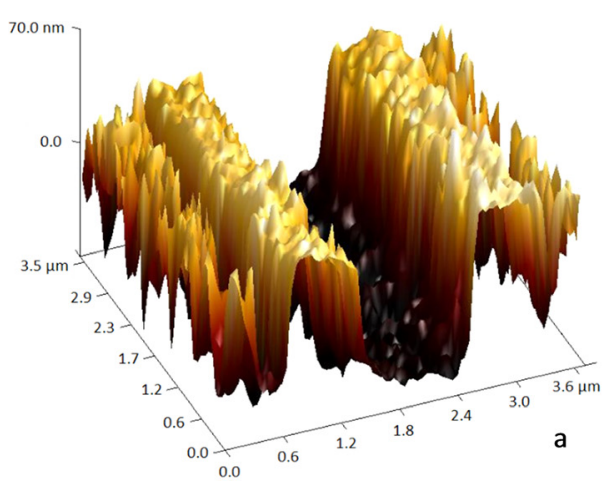

a

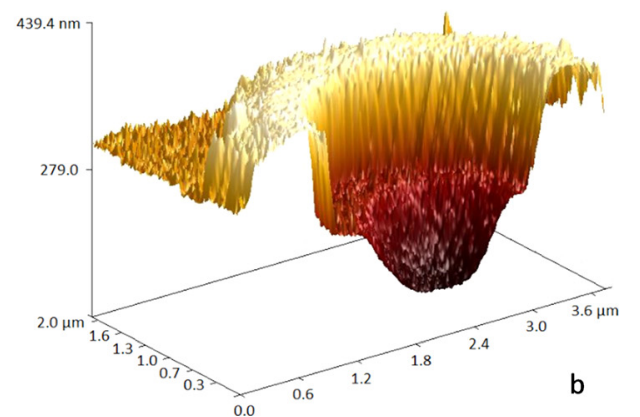

b
FIG. 4. 3D AFM view of topology induced by the combination of DLW and chemical etching for a double plan structure (a) and a cylindrical structure (b). between the planes is highly decreased after the front beam passage.

\section{Surface engineering}

The photo-induced structures, brought to surface by polishing, were immersed in demineralized water for different durations. Selective surface solubility was evidenced and the resulting topology was measured and characterized using AFM, as shown in Figure 4.

Three zones can be distinguished across the section of both structures. The center of the pattern is the most sensitive to chemical etching. The edge of the pattern corresponds to the least etched area, while the pristine zone shows an intermediate etching rate. Pristine glass solubility has been previously studied and had shown that for our condition, the glass surface etching remains below $100 \mathrm{~nm} .{ }^{15}$ After 6 min in demineralized water, a depth variation of about $130 \mathrm{~nm}$ occurs as compared to the non-photo-exposed area. The overlay of the luminescence feature, the silver concentration map, and the water etching is presented in Figure 5.

Chemical selective etching after femtosecond laser exposure has been widely explored in silica glass. In this later case, the femtosecond exposed areas undergo a significant etching that can lead to the fabrication of cavities of several millimeters with high aspect ratio. ${ }^{5,6}$ Correlations between refractive index changes, mechanical stress distributions,

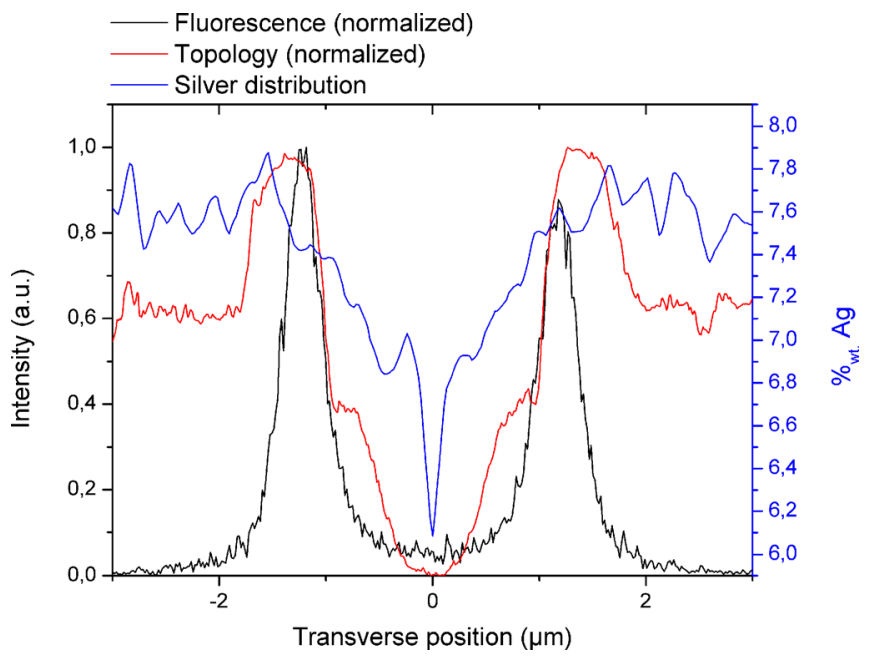

FIG. 5. Superimposition of the fluorescence distribution (black), normalized topology after chemical etching (red), and silver concentration distribution (blue) across the cylindrical structures. nano-grating formation and selective chemical etching have been established in silica.

From a general point of view, the mechanisms at the origin of the selective etching in silicate glass after a pulsed laser interaction are dependent on the glass composition and on the laser regime (irradiance, repetition rate, cumulative deposited dose, and possibly laser polarization). ${ }^{5,16}$ In phosphate based materials, few investigations have been conducted.

The chemical etching is occurring in our case in the whole exposed area with a maximum in the center of the structure, except in the location of the luminescent ring structure. A correlation between the silver concentration and the etching rate has been first considered. Recently, the solubility in de-ionized water of unexposed zinc-phosphate glass has clearly shown that the presence of silver tends to increase the solubility of the glass in water and acid. ${ }^{15}$ In the present case, the lowering of the silver concentration in the center of femtosecond exposed area is not related to a reinforcement of the glass with respect to water etching. Other phenomena have to be taken into account, like stress-induced effects reported in silica which can modify the etching rate. ${ }^{6}$ In phosphate glass, the influence of femtosecond laser has been mainly investigated for refractive index modification. Using $1 \mathrm{kHz}$ repetition rate laser, Bhardwaj et al. reported that negative refractive index variation was observed in SCHOTT IOG-1 phosphate glass without a clear etching rate change. ${ }^{17}$ Ehrt et al. demonstrated in fluoro-phosphate glasses that important positive refractive index variation can be obtained. It is attributed in this particular case to a change of connectivity between fluorine and phosphate groups. ${ }^{18}$ High repetition rate laser can favor ionic migration like in potassium-lanthanum-phosphate glass, where positive index variation was observed following the fluctuations of $(\mathrm{K}, \mathrm{La})$ local concentration. In the present investigation, the generation of hole/ electron pairs leads to the formation of $\mathrm{Ag}^{2+}$ and $\mathrm{Ag}^{0}$ species mainly. Most of the electron traps are gathered in the ring structure to form silver clusters $\mathrm{Ag}_{\mathrm{m}}{ }^{\mathrm{x}+}$ while the chemical nature of the hole centers is still an open discussion. The departure of silver from the center of the structure is expected to lead to compensation mechanisms. No molecular oxygen signature could be evidenced in the center of the structure by Raman spectroscopy mainly due to the remaining weak luminescence that hides the Raman response. One has to consider that the chemical etching could be related to the existence of hole traps and the resulting modification of the glass network by a non-complete compensation of the silver 
depletion. The silver migration and the possible oxygen departure could also result in a decrease of the material density and an increase of the free volume. In silica, several research groups mentioned that stress accumulation and glass densification accelerate the etching rate. ${ }^{19}$ In our case, the use of high repetition rate laser induces local heating near the voxel. However, it is not possible at this stage of the investigation to identify a stress accumulation and an associated selective chemical etching. Regarding the influence of the presence of nano-gratings, since the laser regime used in the present study does not correspond to their formation, the influence of such nano-structure cannot be considered here. ${ }^{5}$

The above description of topological surface structuring is attractive. However, the polishing step to bring motifs to the surface is time-consuming, as well as nontrivial since it is hard (i) to finely preserve the surface orientation with respect to laser-written pattern, and (ii) to control the matter removal at the sub-micron scale. These drawbacks can be solved thanks to direct writing at the glass surface, especially for the engineering of fine multiscale 2D patterns, as shown in Figure 6 after deionized water etching of $10 \mathrm{~min}$ in an ultrasonic bath. However, writing directly at the surface
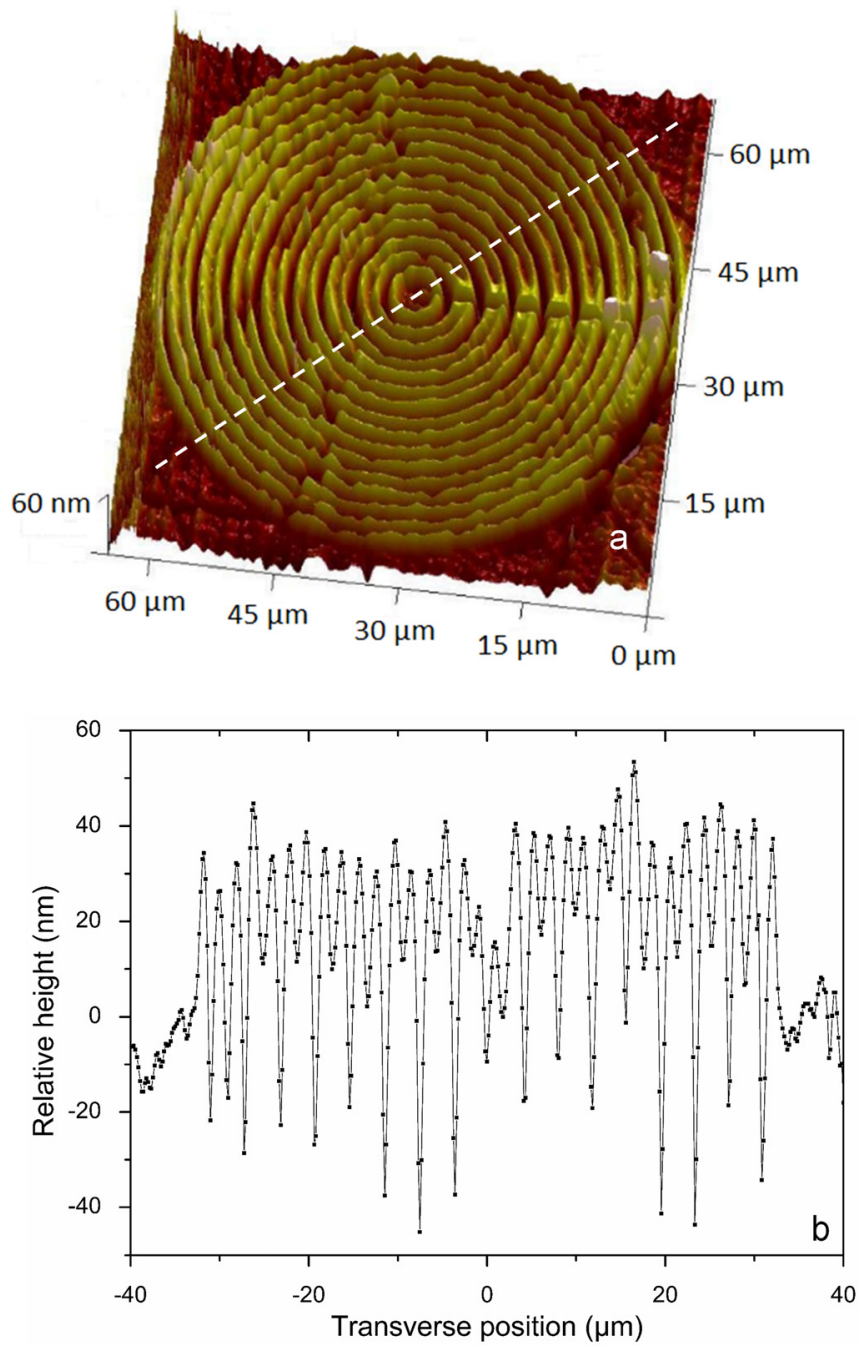

FIG. 6. Surface induced structure thanks to the combination of DLW and DI water etching during $10 \mathrm{~min}$ in an ultrasonic bath (a) and its topology profile along the white dashed line (b). requires extra care, since damage thresholds are much lower. In this framework, surface laser writing of concentric circles was performed slightly above the DLW fluorescence threshold, with a typical irradiance of $3.2 \pm 0.2 \mathrm{TW} / \mathrm{cm}^{2}$. Dose accumulation has been achieved here by multi-scans writing procedure (5 times rescanned).

The cross profile of topology (Figure 6(b)) illustrates that it is also possible to have an accurate control on the design of patterns at the surface, even if such irradiation is more critical than that in the bulk of the glass. Indeed, the revealed topology after etching shows spaces of $2.00 \pm 0.14 \mu \mathrm{m}$ between two peaks, a groove width of $0.75 \pm 0.06 \mu \mathrm{m}$ in the center of the laser interaction zone and $1.00 \pm 0.24 \mu \mathrm{m}$ between two traces. Considering a baseline at the level of the pristine zone, the peaks represent a positive topology of $36.4 \pm 7 \mathrm{~nm}$, the groove depth is $-22.9 \pm 13.2 \mathrm{~nm}$ in the center of the laser interaction zone and $+10.3 \pm 9.7 \mathrm{~nm}$ between two laser traces. Such innovative approach may further be exploited so as to produce 2D photonics structures as Fresnel-type lenses, arrays of antennae or effectively artificial media if smaller lateral dimensions could be achieved.

\section{CONCLUSION}

In summary, it has been shown that femtosecond direct laser writing with high repetition rates produces a silver depletion at the center location of the photo-produced structures. This migration is proposed to be at the origin of the formation of luminescent silver species that can be implemented for sample motions during the DLW process along and perpendicular to the laser propagation axis. Associated to this photo-induced structuring, selective chemical etching in soft conditions has been evidenced in de-ionized water. Lower etching rate has been observed in the location of the fluorescent silver clusters while higher etching rate is clearly observed in the central part of the irradiated zone, leading to multidimensional structures with nanometer-scale controlled depth topology.

\section{ACKNOWLEDGMENTS}

This research was supported by the CNRS, the French Agency of National Research, the French Aquitaine region, the Cluster of Excellence LAPHIA. Confocal imaging was performed at the Photonic Imaging platform of Bordeaux Imaging Center (UMS 420 CNRS). AFM images of Figure 6 were recorded by Fabien Morote at the NIS platform of LOMA.

${ }^{1}$ K. M. Davis, K. Miura, N. Sugimoto, and K. Hirao, Opt. Lett. 21, 1729 (1996).

${ }^{2}$ Q. Sun, F. Liang, R. Vallée, and S. L. Chin, Opt. Lett. 33, 2713 (2008).

${ }^{3}$ A. Stone, M. Sakakura, Y. Shimotsuma, G. Stone, P. Gupta, K. Miura, K. Hirao, V. Dierolf, and H. Jain, Opt. Express 17, 23284 (2009).

${ }^{4}$ S. Kanehira, K. Miura, and K. Hirao, Appl. Phys. Lett. 93, 023112 (2008). ${ }^{5}$ C. Hnatovsky, R. S. Taylor, E. Simova, P. P. Rajeev, D. M. Rayner, V. R. Bhardwaj, and P. B. Corkum, Appl. Phys. A 84, 47 (2006).

${ }^{6}$ Y. Bellouard, A. Said, M. Dugan, and P. Bado, Opt. Express 12, 2120 (2004).

${ }^{7}$ G. Papon, Y. Petit, N. Marquestaut, A. Royon, M. Dussauze, V. Rodriguez, T. Cardinal, and L. Canioni, Opt. Mater. Express 3, 1855 (2013). 
${ }^{8}$ G. Papon, N. Marquestaut, Y. Petit, A. Royon, M. Dussauze, V. Rodriguez, T. Cardinal, and L. J. Canioni, Appl. Phys. 115, 113103 (2014).

${ }^{9}$ A. Royon, K. Bourhis, M. Bellec, G. Papon, B. Bousquet, Y. Deshayes, T. Cardinal, and L. Canioni, Adv. Mater. 22, 5282 (2010).

${ }^{10}$ A. Royon, Y. Petit, G. Papon, M. Richardson, and L. Canioni, Opt. Mater. Express 1, 866 (2011).

${ }^{11}$ N. Marquestaut, Y. Petit, A. Royon, P. Mounaix, T. Cardinal, and L. Canioni, Adv. Funct. Mater. 24, 5824 (2014).

${ }^{12}$ M. Bellec, A. Royon, B. Bousquet, K. Bourhis, M. Treguer, T. Cardinal, M. Richardson, and L. Canioni, Opt. Express 17, 10304 (2009).

${ }^{13}$ M. Bellec, A. Royon, K. Bourhis, J. Choi, B. Bousquet, M. Treguer, T. Cardinal, J. J. Videau, M. Richardson, and L. Canioni, J. Phys. Chem. C 114, 15584 (2010).
${ }^{14}$ K. Bourhis, A. Royon, M. Bellec, J. Choi, A. Fargues, M. Treguer, J. J. Videau, D. Talaga, M. Richardson, T. Cardinal, and L. Canioni, J. Non Cryst. Solids 356, 2658 (2010).

${ }^{15}$ J. Massera, K. Bourhis, L. Petit, M. Couzi, L. Hupa, M. Hupa, J. J. Videau, and T. Cardinal, J. Phys. Chem. Solids 74, 121 (2013).

${ }^{16}$ N. Varkentina, T. Cardinal, F. Morot, P. Mounaix, P. Andre, Y. Deshayes, and L. Canioni, Opt. Express 21, 29090 (2013).

${ }^{17}$ V. R. Bhardwaj, E. Simova, P. B. Corkum, D. M. Rayner, C. Hnatovsky, R. S. Taylor, B. Schreder, M. Kluge, and J. Zimmer, J. Appl. Phys. 97, 083102 (2005).

${ }^{18}$ D. Ehrt, T. Kittel, M. Will, S. Nolte, and A. Tunnermann, J. Non Cryst. Solids 345-346, 332 (2004).

${ }^{19}$ A. Agarwal and M. Tomozawa, J. Non Cryst. Solids 209, 166 (1997). 